\title{
E-Book Planejamento do Ensino: Apoio para a Formação de Estudantes do Curso de Bacharelado e Licenciatura em Enfermagem
}

\author{
Fernanda dos Santos Nogueira Góes, Luciane Sá de Andrade, Adriana Katia Corrêa, Maria \\ Conceição Bernardo de Mello e Souza, Maria José Clapis, Marlene Fagundes Carvalho \\ Gonçalves, Marta Angélica lossi Silva e Rosangela Andrade Aukar de Camargo
}

Escola de Enfermagem de Ribeirão Preto da Universidade de São Paulo

* Autora para correspondência: fersngoes@eerp.usp.br

\section{RESUMO}

Buscou-se aqui relatar o processo de construção de um livro digital sobre planejamento do ensino no contexto da Educação Básica e Profissional em Enfermagem para ser utilizado como recurso auxiliar por alunos de graduação em Enfermagem da Escola de Enfermagem de Ribeirão Preto-USP. Tal iniciativa deu-se a partir do trabalho de um grupo interdisciplinar de docentes que adotou o referencial teórico da problematização, e, para a construção da metodologia empregada, utilizou quatro fases sequenciais: definição do escopo, planejamento, produção e implementação. Para a avaliação do e-book foram convidados oito experts em conteúdo. Na primeira etapa, o grupo de docentes realizou vários encontros, e optou-se pela estruturação seguindo as etapas de um plano de aula. Assim, foi produzido um e-book de onze páginas no qual foram reunidos texto e mídias como links para vídeos armazenados na internet. $\mathrm{Na}$ avaliação de layout e conteúdo, os peritos emitiram opiniões as quais foram incorporadas na versão final do $e-b o o k$. Concluiu-se que desenvolver o livro digital possibilitou a atuação interdisciplinar docente. Além disso, a obra em questão contribui para suprir a lacuna existente de materiais educativos e inovação no processo de ensino-aprendizagem da Licenciatura em Enfermagem, de modo a atender ao novo perfil de alunos que se apresenta.

Palavras-chave: Educação em Enfermagem; Planejamento de Ensino; E-book.

\begin{abstract}
This work reports the construction of an e-book about teaching planning in the context of Basic and Professional Education in Nursing to be used as an auxiliary resource by nursing undergraduate students at Universidade de São Paulo at Ribeirão Preto College of Nursing. This initiative was based on the work of an interdisciplinary group of teachers that adopted the theoretical reference of the problematization and four sequential phases were used: definition of the scope, planning, production and implementation. For evaluation of the e-book eight experts in content were invited. On the first step, the group of teachers held several meetings and chose the structure following the steps of a lesson plan. Thus, an eleven-page e-book was produced in which text and media were gathered as links to videos stored on the internet. In the evaluation of the layout and content, the experts issued opinions that were incorporated into the final version of the e-book. In conclusion, developing the digital material enabled the interdisciplinary work of the teacher and could contribute to supply the existing gap of educational materials and innovation in the teaching process of the undergraduate nursing education in addition to attending to the new profile of students.
\end{abstract}

Keywords: Nursing Education; Teaching Planning; E-book.

\section{Introdução}

As diretrizes curriculares para a formação de profissionais de nível superior apontam a necessidade de os cursos universitários criarem oportunidades para seus estudantes terem acesso a tecnologias digitais de informação e comunicação no seu processo formativo, e também para o seu domínio como instrumento no seu processo de trabalho no exercício profissional JENSEN, GUEDES \& LEITE, 2016). A depender da forma como são inseridas em cursos de enfermagem, essas tecnologias têm revelado um potencial interessante 
para o processo de ensino-aprendizagem (PARIGI et al., 2015).

O curso de Bacharelado e Licenciatura em Enfermagem da Escola de Enfermagem de Ribeirão Preto (EERP) da Universidade de São Paulo (USP) propõe-se a formar enfermeiros para atuarem como docentes na Educação Profissional nos cursos de nível médio voltados a técnicos de enfermagem, bem como para desenvolverem ações de promoção da saúde na Educação Básica. Assim, o curso investe na formação do enfermeiro-professor, renovando metodologias e buscando formas de integrar as diferentes disciplinas. Um dos desafios colocados ao corpo docente do referido curso é a adoção de metodologias ativas que possibilitem a superação da lógica disciplinar de formação (USP/EERP, 2014). Esses desafios estão em consonância com a área de formação de professores da USP.

O modo como os jovens lidam com a tecnologia, especialmente com as tecnologias móveis, é diferente em comparação com a maneira como seus professores universitários foram formados (CECGHETTINI, 2011). Nesse sentido, uma das possibilidades de utilização de recursos tecnológicos no ensino é a adoção de e-books. Tais materiais surgiram especialmente como recurso auxiliar para estimular a leitura, pois sua produção custa, em média, 30\% a menos que o livro impresso (VIRGINIO \& NICOLAU, 2012). Por isso, para a elaboração de um e-book, deve-se considerar a relevância prática dos pontos mais significativos a serem abordados, a fim de que sirva de fato como instrumento auxiliar de aprendizagem, seja na sala de aula ou em outra situação.

Os $e$-books podem utilizar em sua estrutura tanto recursos de hipertexto, como links entre palavras, imagens e elementos gráficos, quanto recursos de hipermídia, incluindo sons, vídeos e animações, de forma a oferecer ao leitor autonomia na fruição do livro (STUMPF et al., 2011).

Assim, tem-se o objetivo de relatar o processo de produção de material digital (e-book) sobre planejamento do ensino no contexto da Educação Básica e Profissional em Enfermagem, a ser utilizado como recurso auxiliar por alunos de graduação em enfermagem, do segundo ao quinto ano do curso de Bacharelado e Licenciatura em Enfermagem da EERP-USP.

O tema "planejamento de ensino" foi escolhido pelo grupo de docentes da EERP-USP, pois se têm inserido no conteúdo programático de disciplinas da área de Educação (Promoção da Saúde na Educação Básica e Educação Profissional em Enfermagem) aspectos ligados à questão do planejamento, entre outras, com o objetivo de preparar o aluno para as atividades docentes, sublinhando a necessidade de o professor planejar, refletir sobre sua ação e a forma como ela se dará, antes durante e depois, afinal o ensino visa à formação para o trabalho e para a vida (LEAL, 2005).

Cabe ressaltar que a produção do material didático digital e a compra de equipamentos para a implementação do produto desenvolvido foram viabilizadas por recurso disponibilizado pela Pró-Reitoria de Graduação da USP para atividades de produção de materiais didáticos, no Edital Pró-Ensino 2012 - Programa de Apoio à Produção de Materiais Didáticos, no qual a EERP-USP foi contemplada com dois recursos distintos.

\section{Método}

Para fins de elaboração do e-book Planejamento de Ensino (GÓES et al., 2015), utilizaram-se um referencial pedagógico para a construção do conteúdo e um referencial metodológico para o desenvolvimento técnico da tecnologia, descritos a seguir.

Foi escolhido o referencial pedagógico da problematização, considerando o aluno como ser político, social, intelectual, livre e autônomo (FREIRE, 2003). Deve-se respeitar a dignidade do sujeito, levando-se em conta que o indivíduo é o ser que constrói a sua própria história. Para tanto, faz-se necessário mudar as perspectivas da sala de aula, criando diferentes oportunidades para que o aluno tenha acesso aos conhecimentos científicos utilizando-se de estratégias de ensino-aprendizagem que levem à apropriação desses conhecimentos.

Para o desenvolvimento tecnológico do material didático, foi usado o modelo proposto 
por Bernardo (1996), composto por quatro fases sequenciais de desenvolvimento: definição do escopo, planejamento, produção e implementação.

A definição do escopo foi realizada a partir de grupos de trabalho formados pelos docentes responsáveis pelo estudo e por dois bolsistas envolvidos. Nessa etapa, utilizou-se a técnica de tempestade cerebral (ANASTASIOU \& ALVES, 2005). A equipe de desenvolvimento de conteúdo foi composta por docentes das áreas de Educação Profissional em Enfermagem e Educação Básica, e se responsabilizou pela elaboração de textos guia, seleção de recursos hipermídia, como áudio, artigos e vídeos do YouTube, com o objetivo de estabelecer um aprendizado interativo. Em seguida foram realizadas a revisão, a atualização e a reordenação contínuas, até a elaboração do conteúdo definitivo.

O planejamento tem como principal meta criar o protótipo completo do material didático digital e o cronograma de execução (BERNARDO, 1996). Foi utilizada multimídia interativa, um formato da tecnologia que combina texto, som, imagens estáticas e vídeo, dispostos de modo a permitir ao usuário controlar os elementos da informação que serão transmitidos (GALVÃO \& PUSGHEL, 2012). A ferramenta de autoria de multimídia empregada foi compatível com aplicativos para sistemas de $\mathrm{iOS} \AA$ e Android $\AA$.

A produção foi realizada por uma equipe técnica contratada para esse fim, a partir da reunião de esboços das telas e do layout final. As mídias foram processadas em programas específicos, a depender de sua natureza (Microsoft Word, CorelDRAW, Aldus PhotoStyler, Microsoft VidEdit, Creative WaveStudio, entre outros). Após a programação, procedeu-se à verificação para a busca e a ajustes em erros de programação, com o objetivo de garantir a sua operacionalidade.

Na implantação, o produto foi disponibilizado para utilização, e seu funcionamento supervisionado pelo setor de informática da EERP-USP (BERNARDO, 1996). A equipe técnica que produziu o e-book, aliada ao setor de informática, realizou os primeiros testes funcionais, e posteriormente o material didático digital foi avaliado por especialistas da área de Enfermagem e Educação, que não participaram do desenvolvimento, com o objetivo de sugerirem possíveis adequações no conteúdo ou no layout.

Para a avaliação, utilizou-se uma amostra de conveniência: foram convidados oito especialistas os quais analisaram o $e$-book em iPads e responderam a um instrumento de avaliação armazenado em banco de dados on-line.

\section{Resultados}

A fim de apresentar os resultados, detalharemos a sequência de construção do $e-b o o k$.

\section{Definição do Escopo}

Na definição do escopo, o grupo interdisciplinar de docentes de enfermagem foi formado por três professores que atuam na área de conhecimento Promoção da Saúde na Educação Básica e cinco professores que atuam na Educação Profissional de nível médio em Enfermagem. O grupo decidiu realizar encontros frequentes para estruturar o conteúdo do material didático digital (e-book), considerando as etapas clássicas de elaboração de uma aula/disciplina: identificação, objetivos educacionais, conteúdos de ensino, métodos e estratégias e avaliação (ZABALA, 2008). Após a definição inicial, formaram-se duplas de docentes para elaborarem partes do texto e selecionarem imagens, vídeos, links da internet, que facilitariam a compreensão do tema, os quais foram apresentados para todo o grupo, sendo escolhidos os mais convenientes e adequados.

Para apresentar o propósito do e-book ao público-alvo (estudantes de graduação), optou-se pela utilização de um vídeo, o qual foi gravado pela equipe técnica de setor de informática do campus da USP de Ribeirão Preto.

\section{Planejamento}

No planejamento, foi feita a opção pelo desenvolvimento do conteúdo em diferentes linguagens computacionais compatíveis para sistemas iOS, Android e Moodle Stoa da USP, e houve a definição de conteúdos e mídias que foram incluídos. 
Para facilitar a utilização do $e$-book pelos estudantes de graduação da EERP, também foi prevista no projeto a compra de dez iPads, nos quais o material educativo foi disponibilizado. Para o uso no ensino, cada docente do grupo de trabalho ficou responsável por, no mínimo, um iPad.

\section{Produção}

A produção foi realizada por uma equipe técnica, contratada para esse fim. Essa equipe reuniu o conteúdo construído pelo grupo de docentes, adequou o layout e realizou ajustes finais após algumas avaliações realizadas pela equipe de desenvolvimento.

\section{Implementação}

O e-book foi disponibilizado via iPads (adquiridos para esse propósito) e publicado na Apple Store e no Moodle Stoa. Para avaliar seu conteúdo e layout antes de ele chegar aos usuários finais, um grupo de oito docentes e doutorandos em Enfermagem navegaram pelo e-book, preencheram um instrumento e expressaram opiniões e sugestões sobre ele, que estão apresentadas abaixo.

Todos os avaliadores elogiaram a produção do material educativo, com ênfase na importância da produção para a Licenciatura em Enfermagem:

Bem didático e traz um conceito bastante importante. Achei ótima a escolha do tema.

Muito dinâmico e muito interessante para usá-lo no dia a dia, quando houver dúvidas. Serve de motivação para estudar, não fica um estudo maçante e cansativo.

Adorei o tema. A abordagem foi ampla e facilitou muito meu aprendizado, pois pretendo seguir a carreira de docente, parabéns!

A estruturação dos temas da forma como estão apresentados permite maior compreensão acerca da temática. Assuntos abordados de forma rica, interessante e interativa.
Muito bom o e-book, facilita o aprendizado pelos alunos de graduação e pós-graduação, levando a uma facilidade de entendimento do que será abordado na aula. Ótima ideia.

As referências utilizadas são de grande relevância e são muito pertinentes para a temática abordada. Os textos ficaram explicativos e as figuras um ótimo recurso.

O uso de recursos multimídia foi enfatizado pelos avaliadores, conforme comentários abaixo:

Mas a parte que me chamou mais atenção sem dúvida foi o uso do vídeo.

Esse recurso é uma alternativa rápida e prática de acesso ao conteúdo a ser estudado. $\mathrm{O}$ aluno tem à sua frente oportunidade de acessar vídeos relacionados e demais ferramentas explicativas.

[...] os demais vídeos vistos, tais como do YouTube, com outros professores também são interessantes e, ao mesmo tempo, permitem outros links na tela ao lado sobre o mesmo assunto. Então o aluno tem mais chances de pesquisar sobre tal conteúdo.

Apresentam-se as sugestões que foram acatadas pelo grupo de produção do e-book e as que não foram seguidas, nos quadros 1 e 2 , respectivamente.

No primeiro semestre de 2016, o e-book foi usado em duas disciplinas do curso de Bacharelado e Licenciatura em Enfermagem da EERP-USP.

Uma disciplina da área de Promoção da Saúde na Educação Básica utilizou os dez iPads em um Laboratório de Prática Pedagógica para a construção do plano de aula. Os alunos demonstraram muito interesse na forma de apresentação do conteúdo e manifestaram a percepção de que foi um instrumento útil para a aprendizagem, tendo solicitado novas oportunidades para manusearem o $e$-book.

Em uma oficina sobre plano de aula, 23 alunos do último ano de graduação que cursavam o estágio curricular na Educação Profissional utilizaram o e-book disponibilizado nos dez iPads. A versão PDF 


\begin{tabular}{|c|c|c|}
\hline \multicolumn{2}{|r|}{ Sugestões } & Modificações realizadas \\
\hline \multirow{2}{*}{ Capa } & Símbolos distorcidos. & Ajustado. \\
\hline & Textos em letra branca. & Cor da caixa de texto modificada. \\
\hline \multirow[b]{2}{*}{ Textos } & Incluir a referência completa. & Incluída. \\
\hline & $\begin{array}{l}\text { Rever a pergunta "O que se deve } \\
\text { saber?", pois está repetida. }\end{array}$ & Uma das repetições foi retirada. \\
\hline Botões & Incluir botão voltar. & Incluído. \\
\hline Conteúdo & $\begin{array}{l}\text { Trazer a dica de alimentação saudável } \\
\text { para a página } 9 \text { - fica mais claro }\end{array}$ & Modificado. \\
\hline Links & $\begin{array}{l}\text { O link da palavra "professor" na } \\
\text { página } 6 \text { refere-se à definição de } \\
\text { "planejamento". }\end{array}$ & Corrigido. \\
\hline
\end{tabular}

Quadro 1 - Sugestões emitidas pelos avaliadores, acatadas pelo grupo de produção do $e$-book, 2015.

\begin{tabular}{l|l|}
\multicolumn{1}{|c|}{ Sugestões } & \multicolumn{1}{c|}{ Justificativa } \\
\hline $\begin{array}{l}\text { Separar os itens do } e-b o o k \text { em páginas diferentes. } \\
\text { Disponibilizar os vídeos como conteúdo } \\
\text { armazenado no } e \text {-book e não em formato de links } \\
\text { para YouTube. }\end{array}$ & $\begin{array}{l}\text { Propriedade intelectual e legal dos } \\
\text { autores dos vídeos. }\end{array}$ \\
\hline
\end{tabular}

Quadro 2 - Sugestões emitidas pelos avaliadores, não acatadas pelo grupo de produção do $e$-book, 2015.

do e-book também foi oferecida no Moodle Stoa USP e, a partir da leitura do portfólio dos alunos, as docentes vêm percebendo que o material educativo tem auxiliado os alunos na construção de seus planos de aula e na compreensão da prática docente.

\section{Discussão}

Embora não garantam, as tecnologias podem aumentar as possibilidades de aprofundamento de conhecimentos que não dependam apenas da relação face a face entre alunos e professores, uma vez que, adequadamente planejado, o ensino pode levar a aprendizagem a ambientes que transcendem a sala de aula (ALONSO, 2005). O corpo docente, por meio de um trabalho conjunto, pode agir no sentido de codificar as mensagens pedagógicas, apresentando-as em diferentes formas, segundo o meio técnico escolhido, com a elaboração de materiais específicos (ALONSO, 2005).

Vislumbra-se que em ambiente de educação auxiliado por materiais didáticos digitais, como o e-book, a enfermagem pode se beneficiar de práticas pedagógicas nas quais o aluno é sujeito de seu aprendizado, facilitando o convívio mútuo (COGO et al., 2010).

O desafio do setor educacional é formar cidadãos competentes na aplicação prática de saberes, capazes de compreenderem o mundo, de se enquadrarem nele e de encontrarem soluções para as situações cotidianas sobre as quais se deve refletir para ressignificar o saber (NOBREGA-THERRIEN et al., 2010). A interação, que pode ser favorecida pela educação mediada pelo computador, fornece subsídios para o crescimento 
e pode ser instrumento de transformação social. Atualmente é quase inconcebível pensar num ensino estritamente tradicional para alunos que têm acesso a todo tipo de recurso e informação desde as séries iniciais da vida escolar, discentes estes que produzem pesquisa e recursos de comunicação e informação em suas atividades escolares (JULIANI \& KURCGANT, 2009).

A utilização da tecnologia no processo de ensino-aprendizagem está plenamente justificada se levar em conta que um dos objetivos básicos da educação é preparar os estudantes para serem cidadãos de uma sociedade plural, democrática e tecnologicamente avançada (MAIZTEGUI et al., 2002). Um dos modos de uso da tecnologia está relacionado com o apoio ao processo de ensino-aprendizagem em atividades presenciais, em situações em que os recursos computadorizados podem tornar conteúdos abstratos e de difícil entendimento em situações mais interessantes e vinculadas à realidade, facilitando o aprendizado e contribuindo com o professor.

Corrobora-se Levy (1999) ao afirmar que não se trata de passar do presencial para a distância ou de estratégias tradicionais para recursos multimídia, mas da transição entre a educação e a formação estritamente institucionalizadas para uma situação de intercâmbio generalizado dos saberes gerenciados pela sociedade, autogeridos, móveis e contextuais, na qual indivíduos, grupos e organizações são considerados recursos potenciais de aprendizado.

\section{Conclusões}

A partir da realização deste projeto foi possível integrar diferentes professores para discussão e consenso de bases teóricas que estruturam o plano de aula. Além disso, a produção desse material educativo digital proporcionou aos discentes conteúdos didáticos relacionados com a formação docente, os quais podem ser disponibilizados em tecnologias móveis, favorecendo o uso e atendendo ao novo perfil de alunos que se apresenta.

Assim, os docentes envolvidos na presente proposta vêm acompanhando, participando ativamente e implementando ações estratégicas que concretizam as políticas de formação propostas, na perspectiva de contribuir para a consolidação do SUS e para o aprimoramento do ensino de graduação em Enfermagem. Com base nessas experiências, foram delineadas as ações propostas neste projeto.

A produção do e-book Planejamento do Ensino, ao considerar as inúmeras possibilidades do uso da tecnologia na educação, colaborou para suprir a lacuna existente devido à escassez de materiais educativos e trouxe inovação ao processo de ensino-aprendizagem da formação em licenciatura em Enfermagem.

O e-book está armazenado em dez iPads, os quais ficam à disposição de docentes e estudantes de Enfermagem. Do mesmo modo o e-book em versão estática pode ser acessado via plataforma Moodle vinculada às disciplinas que ministram conteúdos sobre Educação Profissional em Enfermagem e Educação Básica. Além disso, está disponível no App Store para download. Cabe destacar que o grupo tem investido na sua disponibilização em versão para Android.

\section{Referências Bibliográficas}

ALONSO, Katia M. "Algumas Considerações sobre Educação a Distância, Aprendizagens e a Gestão de Sistemas Não Presenciais de Ensino". In: PRETI, Osvaldo et al. (orgs.). Educasão a Distância: Ressignificando Práticas. Brasília: Liber Livro Editora, 2005.

ANASTASIOU, Lea das Graças Camargo \& ALVES, Leonir Pessate. "Estratégias de Ensinagem". In: Processos de Ensinagem na Universidade: Pressupostos para as Estratégias de Trabalho em Aula. 5. ed. Joinville, SC: Univille, 2005.

BERNARDO, Vinicius. Metodologia para Desenvolvimento de Projeto Multimídia Aplicado ao Ensino da Medicina. Dissertação (Mestrado), Escola Paulista de Medicina, Universidade Federal de São Paulo, São Paulo, 1996.

CECCHET TINI, Eliane. "Introdução". In: VERAS, Marcelo (org.). Inovação e Métodos de Ensino para Nativos Digitais. São Paulo: Atlas, 2011, p. 9.

COGO, Ana Luisa Petersen; SILVEIRA, Denise Tolfo; PEDRO, Eva Neri Rubim et al. "Aprendizagem de Sinais Vitais Utilizando Objetos Digitais: Opinião de Estudantes de Enfermagem". Revista Gaúcha de Enfermagem, Porto Alegre, vol. 31, pp. 435-441, 2010.

FREIRE, Paulo. Pedagogia da Autonomia: Saberes Necessários à Prática Educativa. 28. ed. São Paulo: Paz e Terra, 2003. 
GALVÃO, Elizabeth Correia Ferreira \& PUSCHEL, Vilanice Alves Araújo. "Aplicativo Multimídia em Plataforma Móvel para o Ensino da Mensuração da Pressão Venosa Central". Rev. Esc. Enferm. USP, São Paulo, vol. 46, n. spe, pp. 107-115, 2012.

GÓES, Fernanda dos Santos Nogueira; ANDRADE, Luciane Sa; MISHIMA, Silvana Martins et al. Plano de Aula: Apoio e Fundamentos para Prática Docente - Ebook. Ribeirão Preto, SP: Fierp - Escola de Enfermagem de Ribeirão Preto, 2015, vol. 1, 11 p.

JENSEN, Rodrigo; GUEDES, Erika de Souza \& LEITE, Maria Madalena Januário. "Informatics Competencies Essential to Decision Making in Nursing Management". Rev. Esc. Enferm. USP, São Paulo, vol. 50, n. 1, pp. 109-117, fev. 2016.

JULIANI, Carmen Maria Casquel Monti \& KURCGANT, Paulina. "Tecnologia Educacional: Avaliação de um Web Site sobre Escala de Pessoal de Enfermagem". Rev. Esc. Enferm. USP, São Paulo, vol. 43, n. 3, pp. 512519, set. 2009.

LEAL, Regina Barros. "Planejamento de Ensino: Peculiaridades Significativas". Revista Iberoamericana de Educación, Evora, n. 3, pp. 1-6, 2005.

LEVY, Pierre. Cibercultura. São Paulo: Editora 34, 1999.

MAIZTEGUI, Alberto; ACEVEDO, João A.; CACHAPUZ, Antonio et al. "Papel de la Tecnología en la Educación Científica: una Dimensión Olvidada". Revista Iberoamericana de Educación, n. 28, 2002.
NÓBREGA-THERRIEN, Silvia Maria; GUERREIRO, Maria das Graças da Silva; MOREIRA, Thereza Maria Magalhães et al. "Projeto Político Pedagógico: concepção, construção e avaliação na enfermagem". Rev. Esc. Enferm. USP, São Paulo, vol. 44, n. 3, pp. 679686, 2010.

PARIGI, Dayane Mayara Gambini; TOMAZELLI, Priscila Caccer; ALMEIDA, Denise Maria de et al. "Construção da Identidade Docente na Formação de Professores de Enfermagem: Reflexão Mediada por Tecnologias Digitais". Rev. Esc. Enferm. USP, São Paulo, vol. 49, n. spe2, pp. 144-149, 2015.

STUMPF, Alexsandro; GONÇALVES, Berenice Santos; PEREIRA, Alice Teresinha Cybis \& GONÇALVES, Marilia Matos. "O Livro Digital em Ambientes Virtuais de Aprendizagem: Utilização da Hipermídia como Novas Possibilidades de Leitura". $5^{\circ}$ Conahpa, Congresso Nacional de Ambientes Hipermídia para Aprendizagem. Pelotas, RS, 2011.

UNIVERSIDADE DE SÃO PAULO. Escola de Enfermagem de Ribeirão Preto, EERP-USP. Projeto Pedagógico do Curso de Bacharelado e Licenciatura em Enfermagem da Escola de Enfermagem de Ribeirão Preto, 2014.

VIRGINIO, Rennam \& NICOLAU, Marcos. "Livro Digital: Percalços e Artimanhas de um Mercado em Reconfiguração". Revista Temática, Paraíba, 2012.

ZABALA, A. A Prática Educativa: Como Ensinar. Porto Alegre: Artmed, 2008.

Publicado em 30/06/2017. 